\title{
Características clínicas y microbiológicas de infecciones por Klebsiella pneumoniae productora de carbapenemasa MBL, tipo NDM, Hospital Geriátrico San Isidro Labrador EsSalud, 2018
}

Victor Manuel Buendia Sotelo* 1,a ; Shirley Morales Gutiérrez ${ }^{1, b}$; Jaime Coca Núñez ${ }^{1,2, c}$; Lenin Santos Varas ${ }^{1,3, d}$; José A. Soriano Toyama ${ }^{1}$

\section{RESUMEN}

Objetivo: Determinar las características clínico-microbiológicas de 7 casos de infección nosocomial por Klebsiella pneumoniae productora de carbapenemasa MBL, tipo NDM, en el Hospital Geriátrico San Isidro Labrador (HG SIL) en el periodo de febrero a mayo de 2018.

Materiales y métodos: Es un estudio descriptivo y retrospectivo realizado en adultos mayores con infecciones urinarias, neumonías, úlceras sacras e infecciones de herida operatoria que recibieron terapia antibiótica múltiple no protocolizada. El método usado para detectar la MBL fue el de doble disco con ácido fenilborónico (APB) y ácido etilendiaminotetraacético (EDTA) en los aislados de Klebsiella pneumoniae con sensibilidad disminuida a carbapenémicos. La identificación del gen NDM se realizó en el Instituto Nacional de Salud mediante reacción en cadena de la polimerasa.

Resultados: En total, fueron 7 casos positivos para el gen NDM y todos resistentes al meropenem lo que confirmó el brote epidémico. La mortalidad fue 28,6 \% (2 de 7 pacientes) pero, debido a la existencia de comorbilidades en todos los casos, no se pudo determinar la mortalidad atribuible.

Conclusiones: Destacan el rol clave inicial del laboratorio para detectar y tipificar las carbapenemasas y las medidas de control integral de las infecciones. Además, son los primeros casos reportados en nuestra red asistencial.

Palabras clave: Infecciones por Klebsiella; Enterobacteriaceae resistentes a los carbapenémicos; Resistencia betalactámica (Fuente: DeCS BIREME).

\section{Clinical and microbiological characteristics of New Delhi MBL (NDM)-producing Klebsiella pneumoniae infections at the Hospital Geriátrico San Isidro Labrador EsSalud, 2018}

\section{ABSTRACT}

Objective: To determine the clinical and microbiological characteristics of seven (7) cases of New Delhi MBL (NDM)producing Klebsiella pneumoniae nosocomial infections at the Hospital Geriátrico San Isidro Labrador (HG SIL) from February to May 2018.

Materials and methods: A descriptive and retrospective study conducted in elderly people with urinary infections, pneumonia, sacral ulcers and surgical wound infection who received multiple non-protocolized antibiotic therapy. The methods used to detect MBLs in the Klebsiella pneumoniae isolates that showed decreased sensitivity to carbapenems were the phenylboronic acid-based (PBA) and ethylenediaminetetraacetic acid-based (EDTA) double disk tests. The identification of the NDM gene was performed by polymerase chain reaction at the Instituto Nacional de Salud.

Results: In total, the seven cases were positive for the NDM gene and resistant to meropenem, which confirmed the epidemic outbreak. Mortality accounted for $28.6 \%$ ( 2 of 7 patients) of the cases. However, due to the presence of comorbidities in all cases, the attributable mortality could not be determined.

Conclusions: Labs have a key initial role for detecting and classifying the carbapenemases and the measures of comprehensive control of infections. The aforementioned cases are the first ones reported in our healthcare network.

Keywords: Klebsiella infections; Carbapenem-Resistant enterobacteriaceae; Beta-Lactam resistance (Source: MeSH NLM).

1 Hospital Geriátrico San Isidro Labrador, Red Prestacional Almenara-EsSalud. Lima, Perú.

2 Universidad Peruana Cayetano Heredia Facultad de Medicina. Lima, Perú.

3 Universidad Mayor de San Marcos. Lima, Perú.

a Master en Gerencia de servicios de Salud, egresado del Doctorado de salud pública.

b Postgrado Universidad Federico Villarreal.

c Especialista en Microbiología.

d Tecnólogo médico.

* Autor corresponsal

Horiz Med (Lima) 2020; 20(2): e915 


\section{INTRODUCCIÓN}

Las infecciones intrahospitalarias, catalogadas como infecciones asociadas a la atención de salud (IAAS), son un problema de salud pública en todo el mundo por la multirresistencia bacteriana generada a varios antimicrobianos de última generación, lo que deviene en una elevada mortalidad e incremento significativo de los costos de atención. Estos efectos constituyen un serio desafío para las instituciones prestadoras de servicios salud y también para el paciente, su familia, la comunidad y el Estado ${ }^{(1,2)}$.

Las IAAS se presentan en países desarrollados y en desarrollo. Se estima que 1,4 millones de pacientes adquieren una infección nosocomial diariamente, y que dos millones adquirirán una infección intrahospitalaria cada año durante su estadía en establecimientos de Estados Unidos de Norteamérica y, de ellos, cerca de 90000 morirán debido a estas infecciones ${ }^{(3)}$.

Desde 2007, en el grupo de los productores de metalobetalactamasa (MBL), han aparecido unos microorganismos con un mecanismo de resistencia especial, denominado Nueva Delhi metalobetalactamasa (NDM), que son resistentes a todos los antibióticos betaláctamicos, excepto al aztreonam ${ }^{(4)}$. En el 2011, este mecanismo se detectó en Guatemala ${ }^{(5)}$ y dos años después, en Colombia ${ }^{(6)}$. Al año siguiente, la Organización Panamericana de la Salud (OPS) y la Organización Mundial de la salud (OMS) reforzaron sus recomendaciones para las IAAS y destacaron la importancia de establecer medidas de prevención oportunas y control de la infección en los establecimientos de salud, así como para la vigilancia y detección de este mecanismo de resistencia.

En 2016, en un hospital de Cusco (Perú) se reportaron tres casos de $K$. pneumoniae resistente a carbapenémicos. $\mathrm{El}$ mecanismo de resistencia no se identificó pero estaría mediado por enzimas tipo carbapenemasas, principalmente, del tipo Klebsiella pneumoniae carbapenemasa (KPC) o NDM, que ya estarían en nuestro país desde hace algunos años en condiciones de subrregistro, por la falta de experticia $y$ de medios de identificación ${ }^{(7)}$. Estas infecciones tienen una mortalidad global mayor de $50 \%$, aunque en el Perú alcanzaron el $55 \%$ en una serie de nueve casos ${ }^{(8)}$.

El Hospital Geriátrico San Isidro Labrador (HGSIL) de EsSalud es un establecimiento de nivel II-E de mediana estancia para la población adulta mayor referida de los establecimientos de salud pertenecientes a la Red Desconcentrada Almenara. Su principal proveedor de pacientes es el Hospital Nacional Guillermo Almenara Irigoyen (HNGAI), en un porcentaje que oscila entre 54,4 a $58,5 \%{ }^{(9)}$. Desde el 2016 se ha reportado la presencia de bacilos gramnegativos productores de carbapenemasas ${ }^{(10)}$, que serían Escherichia coli y Klebsiella pneumoniae resistentes a carbapenémicos, pero hay una escasa evidencia para su diagnóstico clínico y manejo oportuno.

Entre febrero y abril de 2018 se detectaron en este hospital siete casos de infección por Klebsiella pneumoniae MBL, subtipo NDM. Estos pacientes fueron referidos a nuestro centro asistencial desde el HNGAl con otros diagnósticos para continuar sus tratamientos. En el presente estudio se describen las características clínicas y microbiológicas de los casos detectados y confirmados por estudio molecular (reacción en cadena de la polimerasa o PCR, de sus siglas en inglés) en el Instituto Nacional de salud (INS) que representan los primeros casos identificados y reportados en el Seguro Social (EsSalud).

\section{MATERIALES Y MÉTODOS}

\section{Diseño y población de estudio}

Estudio descriptivo y retrospectivo de 7 casos de infección por Klebsiella pneumoniae MBL tipo NDM durante su hospitalización en el HGSIL en el periodo de febrero a mayo de 2018. El recojo y manejo de datos se realizó luego del control del brote epidémico. Se revisaron las historias clínicas y los registros de laboratorio de los pacientes clasificados de infección por K. pneumoniae.

\section{Variables y mediciones}

Para definir un caso infectado se consideraron los siguientes aspectos clínicos y microbiológicos registrados en las historias clínicas: procedencia del HNGAl, fiebre $>38^{\circ} \mathrm{C}$, escalofríos, malestar general, taquicardia, herida infectada, leucocitosis con desviación izquierda, proteínaC-reactiva mayor de $10 \mathrm{mg} / \mathrm{L}$ y cultivo positivo a Klebsiella pneumoniae MBL, NDM en fuente seleccionada. Las muestras se sembraron en agares sangre, Mac Conkey y chocolate. La identificación y sensibilidad bacteriana se realizó en el analizador microbiológico modelo MicroScan (autoSCAN-4 System Beckman Coulter ${ }^{\circledR}$ ). En las cepas con sensibilidad disminuida a carbapenémicos, las carbapenemasas se detectaron con el método de la inactivación del meropenem y sinergia de doble disco con ácido fenilborónico y ácido etilendiaminotetraacético (EDTA). El gen NDM se identificó con la reacción en cadena de la polimerasa convencional en el Instituto Nacional de Salud (laboratorio de referencia).

\section{Análisis estadístico}

Para el análisis estadístico se calcularon las frecuencias y porcentajes de las variables. Se empleó el programa SPSS v 23 para el análisis descriptivo de las medidas de tendencia central y dispersión.

\section{Consideraciones éticas}

El presente estudio fue evaluado y aprobado por el Comité de Ética en Investigación del HNGAI (carta N ${ }^{\circ} 038$-CIEI-OCID HNGAI del 24-08-2018 y con NIT: 753-2018-1474). 
Características clínicas y microbiológicas de infecciones por Klebsiella pneumoniae productora de carbapenemasa MBL, tipo NDM, Hospital Geriátrico San Isidro Labrador EsSalud, 2018

\section{RESULTADOS}

Por tratarse de un estudio descriptivo y retrospectivo de una serie de 7 casos infectados por Klebsiella pneumoniae MBL tipo NDM presentamos las características clínicas y microbiológicas encontradas en cada uno de estos casos (Tabla 1):

\section{Caso.$^{\circ} 1$}

Paciente varón de 90 años, casado, procedente del servicio de Ortopedia y Traumatología del HNGAl, posoperado de fractura de cadera derecha y trauma uretral, portador de sonda vesical. Había recibido ertapenem, ceftriaxona, imipenem y vancomicina. Ingresó al hospital el 19-05-2018 para continuar tratamiento. Presentó con fiebre $>38^{\circ} \mathrm{C}$, malestar general, taquicardia (100-120 por $\mathrm{min})$, leucocitosis $\left(10280 / \mathrm{mm}^{3}\right)$ y proteína C-reactiva: $145 \mathrm{mg} / \mathrm{L}$. Se aisló en orina Klebsiella pneumoniae BLEE positivo, productora de carbapenemasa confirmado como tipo NDM en INS. Evolucionó con cirrosis hepática, úlcera sacra, insuficiencia renal, septicemia. Recibió vancomicina, meropenen y colistina. Falleció luego de 28 días de hospitalización. El microorganismo aislado fue resistente al meropenem, imipenem y ertapenem, pero sensible a la amicacina, colistina, levofloxacina y nitrofurantoína.

\section{Caso . $^{\circ} 2$}

Mujer de 92 años, casada, procedente del servicio de Medicina del HNGAl con cirrosis hepática, encefalopatía, dismovilidad, sin antibioticoterapia previa. Ingresó el 19-04-2018 para continuar tratamiento. Estuvo hipotérmica, con escalofríos, malestar general, taquicardia 110 por minuto, leucocitosis en $10050 / \mathrm{mm}^{3}$ y proteína C-reactiva: $182 \mathrm{mg} / \mathrm{L}$. En el aspirado endotraqueal se aisló Klebsiella pneumoniae BLEE (betalactamasa de espectro expendido) positiva, productora de carbapenemasa confirmado como tipo NDM en INS. Presentó neumonía, septicemia, oliguria. Recibió imipenem, ceftriaxona, y ciprofloxacina. Falleció luego de 11 días de hospitalización. La bacteria aislada fue resistente al meropenem, imipenem y ertapenem, sensible a la amicacina y la colistina, e intermedio a la levofloxacina.

Caso . $^{\circ} 3$

Varón de 77 años, casado, proviene de Cirugía General y UCI (HNGAI). Posoperado de estrechez uretral y con sonda Foley, traqueotomizado, con enfermedad de Parkinson y úlcera sacra por presión. Había recibido meropenem y vancomicina. Ingresó el 06-02-2018 para continuar la antibioticoterapia. Cursó con fiebre de $38{ }^{\circ} \mathrm{C}$, malestar general, taquicardia 96 por minuto, leucocitos en $7000 \times \mathrm{mm}^{3}$. En la secreción de herida sacra aislaron Klebsiella pneumoniae BLEE positivo, productora de carbapenemasa confirmado como tipo NDM en INS en secreción de herida sacra. Recibió vancomicina y levofloxacina. Luego de 20 días de hospitalización, salió de alta mejorado y con hemocultivo negativo. El microorganismo aislado fue resistente a meropenem, pero sensible a amicacina, colistina, cefalotina, cefoxitina, ciprofloxacina y tigeciclina.

\section{Caso $n .^{\circ} 4$}

Varón de 79 años, casado, procedente del servicio de Cirugía de Esófago y Estómago (HNGAl), postgastrectomizado por neoplasia gástrica, con infección de herida operatoria e hipertrofia prostática. Recibió ciprofloxacina e ingresó al hospital el 27-02-2018 para continuar antibioticoterapia. Evolucionó con febrícula de $37,5{ }^{\circ} \mathrm{C}$, escalofríos, malestar general, taquicardia 90 por minuto, leucocitosis $10410 / c c, y$ proteína C-reactiva $256 \mathrm{mg} / \mathrm{L}$. Se aisló en secreción de herida operatoria Klebsiella pneumoniae BLEE positivo, productora de carbapenemasa confirmado como tipo NDM en INS. Recibió imipenem, amicacina, levofloxacina y clindamicina. Sale de alta mejorado, luego de 22 días de hospitalización. La bacteria aislada fue resistente a meropenem, imipenem, y ertapenem, pero sensible a amicacina y colistina.

\section{Caso $n .^{\circ} 5$}

Varón de 59 años, conviviente, procedente del Servicio de Neurología del HNGAI, sin antibioticoterapia previa, con enfermedad cerebro vascular isquémica, síndrome confusional agudo e hipertensión arterial. Ingresó a este hospital el 04-04-2018 para rehabilitación y tratamiento clínico. Presentó fiebre de $38^{\circ} \mathrm{C}$, escalofríos, malestar general, taquicardia 98 por minuto, leucocitos en $7990 \times \mathrm{mm}^{3}$ y proteína C-reactiva en $72,9 \mathrm{mg} / \mathrm{L}$. Cursó con infección del tracto urinario y se aisló en orina Klebsiella pneumoniae BLEE positiva, productora de carbapenemasa confirmado como tipo NDM en INS. Recibió meropenem y vancomicina. A los 20 días de hospitalización, sale de alta mejorado. El microrganismo aislado fue resistente al meropenem, imipenem, y ertapenem, pero sensible a la amicacina, colistina, levofloxacina, nitrofurantoína y tigeciclina.

\section{Caso n. $^{\circ} 6$}

Mujer de 87 años, viuda, procedente del Servicio de Medicina del HNGAl, portadora de neoplasia de vesícula biliar metastásica al hígado, hipertensa, con hernia epigástrica y dependiente total en sus necesidades. Había recibido clindamicina e imipenen. Ingresó a este hospital el 06-03-2018 para tratamiento paliativo. Presentó febrícula de $37,8{ }^{\circ} \mathrm{C}$, malestar general, taquicardia 98 por minuto, leucocitosis en $11940 \times \mathrm{mm} 3$ y proteína C-reactiva: $72 \mathrm{mg} / \mathrm{L}$. Cursó con deterioro cognitivo y requirió sonda Foley. En la orina se aisló Klebsiella pneumoniae BLEE positivo, productora de carbapenemasa confirmado como tipo NDM en INS. Se trató la infección urinaria con ceftriaxona. Sale de alta, ya mejorada, a los 11 días de hospitalización. El microorganismo aislado fue resistente al meropenem y ertapenem, pero sensible a imipenem, amicacina, colistina, levofloxacina y tigeciclina.

\section{Caso n. $^{\circ} 7$}

Mujer de 81 años, soltera, procedente del servicio de 
Cirugía de Esófago y Estomago del HNGAI, operada de neoplasia gástrica grado II (que se complicó con síndrome pilórico posoperatorio), con desnutrición moderada y sin antibioticoterapia previa. Ingresó a este hospital el 23-03-2018 para continuar tratamiento. Presentó fiebre $38^{\circ} \mathrm{C}$, malestar general. taquicardia 94 por minuto, leucocitosis en $17000 \mathrm{~mm}^{3}$ y proteína C-reactiva $12,4 \mathrm{mg} / \mathrm{l}$. Estuvo intubada en $\mathrm{UCl}$ y en la secreción endotraqueal se aisló Klebsiella pneumoniae BLEE positivo, productora de carbapenemasa confirmada como tipo NDM en INS, por lo que había recibido ceftriaxona y metronidazol. Sale de alta mejorada a los 12 días de hospitalización. El microorganismo aislado fue resistente al meropenen, imipenem y ertapenem. pero sensible a la amicacina, colistina, levofloxacina y tigeciclina.

Del total de pacientes adultos mayores estudiados, 4 eran hombres y 3 mujeres, con una edad media de 80 años y rango de 77 a 92 años. Todos los pacientes procedieron del HNGAI, donde estuvieron hospitalizados en los servicios de Emergencia, Ortopedia y Traumatología, Medicina, $\mathrm{UCl}$, Cirugía General, Cirugía de Esófago y Estómago y
Neurología (Tabla 1).

En 2 casos se diagnosticaron infecciones del tracto urinario (ITU), 2 septicemias, 1 úlcera sacra infectada, 1 infección de herida operatoria y 1 neumonitis aspirativa. En todos los casos se presentaron comorbilidades. Las fuentes de aislamiento de Klebsiella pneumoniae fueron orina $(42 \%)$, secreción de herida $(28,5 \%)$ y aspirado bronquial $(28 \%)$. Cuatro pacientes habían recibido tratamiento antibiótico previo (57 \%) que incluyó cefalosporinas de tercera y cuarta generación. El promedio de permanencia hospitalaria fue de 18 días (desviación estándar: 6,1 días) con un rango de 11 a 28 días.

Los dos pacientes fallecidos por sepsis presentaron hepatopatía crónica como antecedente y comorbilidad. Todos los casos recibieron tratamiento con antibiótico, entre ellos destacan las asociaciones entre carbapenémicos y quinolonas. Asimismo, en todos los casos, el mecanismo de resistencia bacteriano fue la acción de enzimas productoras de MBL y betalactamasa de espectro extendido (BLEE).

Tabla 1. Características de los pacientes infectados por Klebsiella pneumoniae productora de carbapenemasa MBL y NDM en el Hospital San Isidro Labrador. EsSalud 2018.

\begin{tabular}{|c|c|c|c|c|c|c|c|c|c|c|}
\hline 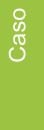 & 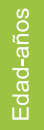 & Sexo & $\begin{array}{l}\text { Procedecia } \\
\text { HNGAI } \\
\text { y servicio }\end{array}$ & $\begin{array}{l}\text { Diagnóstico } \\
\text { de infección }\end{array}$ & Comorbilidad & $\begin{array}{l}\text { Fuente de } \\
\text { de aislamiento }\end{array}$ & $\begin{array}{l}\text { Tratamiento } \\
\text { antibiótico previo } \\
\text { en hospital de origen }\end{array}$ & $\begin{array}{l}\text { Días de } \\
\text { estancia } \\
\text { hospitalaria }\end{array}$ & Mortalidad & $\begin{array}{l}\text { Tratamiento } \\
\text { antibiótico }\end{array}$ \\
\hline 1 & 90 & $M$ & $\begin{array}{l}\text { Trauma- } \\
\text { tología }\end{array}$ & $\begin{array}{l}\text { Septicemia } \\
\text { por ITU }\end{array}$ & $\begin{array}{l}\text { Posoperado } \\
\text { fractura } \\
\text { de cadera } \\
\text { derecha. } \\
\text { Hepatopatía } \\
\text { crónica }\end{array}$ & Orina & $\begin{array}{l}\text { Ertapenem } \\
\text { Ceftriaxona } \\
\text { Clindamicina } \\
\text { Imipenem }\end{array}$ & 28 & sí & $\begin{array}{l}\text { Vancomicina } \\
\text { Meropenem } \\
\text { Colistina }\end{array}$ \\
\hline 2 & 92 & $F$ & Medicina & $\begin{array}{l}\text { Septicemia } \\
\text { por } \\
\text { neumonía }\end{array}$ & $\begin{array}{l}\text { Cirrosis } \\
\text { hepática }\end{array}$ & $\begin{array}{l}\text { Aspirado } \\
\text { endotraqueal }\end{array}$ & No & 11 & sí & $\begin{array}{l}\text { Imipenem } \\
\text { Ciprofloxacina } \\
\text { Ceftriaxona }\end{array}$ \\
\hline 3 & 77 & M & $\begin{array}{l}\text { UCI } \\
\text { Cirugía } \\
\text { General }\end{array}$ & $\begin{array}{l}\text { Úlcera } \\
\text { sacra } \\
\text { infectada } \\
\text { grado IV }\end{array}$ & $\begin{array}{l}\text { Enf } \\
\text { Parkinson, } \\
\text { Traqueosto- } \\
\text { mía, } \\
\text { Sonda } \\
\text { Foley } \\
\text { HTA } \\
\text { HPB }\end{array}$ & $\begin{array}{l}\text { Secreción de } \\
\text { herida sacra }\end{array}$ & $\begin{array}{l}\text { Meropenem } \\
\text { Vancominica }\end{array}$ & 20 & $\begin{array}{l}\text { NO } \\
\text { alta }\end{array}$ & $\begin{array}{l}\text { Vancomicina } \\
\text { Levofloxacina }\end{array}$ \\
\hline 4 & 79 & M & $\begin{array}{l}\text { Cirugía de } \\
\text { Esófago y } \\
\text { Estómago }\end{array}$ & $\begin{array}{l}\text { Infección } \\
\text { de herida } \\
\text { operatoria } \\
\text { post } \\
\text { gastrecto- } \\
\text { mía }\end{array}$ & NM gástrica & $\begin{array}{l}\text { Secreción } \\
\text { de Hda } \\
\text { operatoria }\end{array}$ & Ciprofloxacina & 22 & $\begin{array}{l}\text { NO } \\
\text { alta }\end{array}$ & $\begin{array}{l}\text { Imipenem } \\
\text { Amikacina } \\
\text { Levofloxacina } \\
\text { Clindamicina }\end{array}$ \\
\hline 5 & 59 & M & Neurología & $\begin{array}{l}\text { ITU } \\
\text { nosocomial }\end{array}$ & $\begin{array}{l}\text { ECV } \\
\text { hemorrágico } \\
\text { HTA } \\
\text { Demencia }\end{array}$ & Orina & No & 20 & $\begin{array}{l}\text { NO } \\
\text { Alta }\end{array}$ & $\begin{array}{l}\text { Meropenem } \\
\text { Vancomicina }\end{array}$ \\
\hline 6 & 87 & $\mathrm{~F}$ & $\begin{array}{l}\text { Medicina } \\
\text { Interna. }\end{array}$ & $\begin{array}{l}\text { ITU } \\
\text { nosocomial }\end{array}$ & $\begin{array}{l}\text { Neo Biliar. } \\
\text { Colelitiasis, } \\
\text { HTA, } \\
\text { Sobrepeso }\end{array}$ & Orina & $\begin{array}{l}\text { Clindamicina } \\
\text { Imipenem }\end{array}$ & 14 & $\begin{array}{l}\text { NO } \\
\text { Alta }\end{array}$ & Ceftriaxona \\
\hline
\end{tabular}


Características clínicas y microbiológicas de infecciones por Klebsiella pneumoniae productora de carbapenemasa MBL, tipo NDM, Hospital Geriátrico San Isidro Labrador EsSalud, 2018

\begin{tabular}{|c|c|c|c|c|c|c|c|c|c|}
\hline 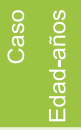 & Sexo & $\begin{array}{l}\text { Procedecia } \\
\text { HNGAI } \\
\text { y servicio }\end{array}$ & $\begin{array}{l}\text { Diagnóstico } \\
\text { de infección }\end{array}$ & Comorbilidad & $\begin{array}{l}\text { Fuente de } \\
\text { de aislamiento }\end{array}$ & $\begin{array}{l}\text { Tratamiento } \\
\text { antibiótico previo } \\
\text { en hospital de origen }\end{array}$ & $\begin{array}{l}\text { Dias de } \\
\text { estancia } \\
\text { hospitalaria }\end{array}$ & Mortalidad & $\begin{array}{l}\text { Tratamiento } \\
\text { antibiótico }\end{array}$ \\
\hline 781 & $\mathrm{~F}$ & $\begin{array}{l}\text { Medicina } \\
\text { Interna. } \\
\text { Serv de } \\
\text { Esófago y } \\
\text { Estómago }\end{array}$ & $\begin{array}{l}\text { Neumonitis } \\
\text { aspirativa }\end{array}$ & $\begin{array}{l}\text { Post. Neo } \\
\text { gástrico, } \\
\text { sin pilórico, } \\
\text { HTA }\end{array}$ & $\begin{array}{l}\text { Aspirado } \\
\text { endotraqueal }\end{array}$ & No & 12 & $\begin{array}{l}\text { NO } \\
\text { Alta }\end{array}$ & $\begin{array}{l}\text { Ceftriaxona } \\
\text { Metronidazol }\end{array}$ \\
\hline
\end{tabular}

HNGAI: Hospital Nacional Guillermo Almenara; ITU: Infección del tracto urinario.

UCI: Unidad de Cuidados Intensivos, HTA: Hipertensión arterial, HPB: Hipertrofia prostática benigna NM: Neoplasia, ECV: Enfermedad cerebrovascular.

Sobre la resistencia antibiótica, se observó que el patrón de resistencia al meropenem alcanzó al $100 \%$ de cepas.

Para el caso del imipenem y el ertapenem la resistencia encontrada fue de $71 \%$ y $86 \%$, respectivamente (Tabla 2 ).

Tabla 2. Patrón de resistencia de los carbapenémicos en la infección por Klebsiella pneumoniae productora de carbapenemasa MBL y NDM en el Hospital San Isidro Labrador. EsSalud 2018

\begin{tabular}{ccccccc} 
& & Meropenem & & Imipenem & & Ertapenem \\
\hline Caso & CIM & Interpretación & CIM & Interpretación & CIM & Interpretación \\
\hline 001 & 4 & $\mathrm{R}$ & 4 & $\mathrm{R}$ & $>1$ & $\mathrm{R}$ \\
\hline 002 & 8 & $\mathrm{R}$ & 8 & $\mathrm{R}$ & $>1$ & $\mathrm{R}$ \\
003 & 4 & $\mathrm{R}$ & $\leq 1$ & $\mathrm{~S}$ & $\leq 0,5$ & $\mathrm{~S}$ \\
004 & $>8$ & $\mathrm{R}$ & 4 & $\mathrm{R}$ & $>1$ & $\mathrm{R}$ \\
005 & 8 & $\mathrm{R}$ & 8 & $\mathrm{R}$ & $>1$ & $\mathrm{R}$ \\
006 & $>8$ & $\mathrm{R}$ & $\leq 1$ & $\mathrm{~S}$ & $>1$ & $\mathrm{R}$ \\
007 & $>8$ & $\mathrm{R}$ & 8 & $\mathrm{R}$ & $>1$ & $\mathrm{R}$ \\
\hline $\begin{array}{c}\text { Resistencia } \\
\mathrm{n}(\%)\end{array}$ & & $7(100 \%)$ & & $5(71 \%)$ & & $6(86 \%)$ \\
\hline
\end{tabular}

Respecto al perfil de susceptibilidad a los antibióticos no carbapenémicos de cepas de Klebsiella pneumoniae, se aprecia que, en todos los casos, las cepas fueron sensibles a la amicacina con un CIM de $\leq 16$ y a la colistina con un $\mathrm{CIM} \leq 2$. Todas las cepas fueron resistentes a la amoxicilina/ ácido clavulánico, ampicilina/sulbactan, aztreonam, cefepima, cefoxitina, cefuroxima, gentamicina, piperazilina/ tazobactam, tobramicina y trimetropim/sulfametoxazol.

\section{DISCUSIÓN}

Los 7 casos de infecciones por Klebsiella pneumoniae descritos en este estudio son los primeros reportados en nuestro hospital, en la Red Desconcentrada Almenara y en EsSalud, pero el segundo reporte a nivel nacional después de los primeros casos reportados en el Hospital Dos de Mayo, Lima ${ }^{(8)}$. Los casos del Hospital Dos de Mayo fueron, en total, 9 (4 varones y 5 mujeres). De ellos, 5 estaban infectados y 4 colonizados, hospitalizados en $\mathrm{UCl}$, la mayoría eran neuroquirúrgicos, con edad media de 56 años, mortalidad global de 55,5 \% y tiempo de hospitalización prolongado. Todos los casos presentaron comorbilidades. Las capas aisladas fueron BLEE (+) y, en todos los casos, hubo susceptibilidad antimicrobiana a la amicacina (CIM $\leq 18)$, hallazgo semejante a lo descrito en el nuestro estudio.

La infección predominante fue la del tacto urinario (42 \%) que, en un caso, derivó en septicemia mortal. Un resultado similar se encontró en 4 de 16 casos reportados de Klebsiella pneumoniae productora de carbapenemasas en el Hospital Virgen del Camino-Pamplona ${ }^{(11)}$. Los siete casos presentaron comorbilidad variable, sin embargo, llama la atención la hepatopatía crónica en los dos casos de pacientes fallecidos.

Las otras fuentes de aislamiento en donde se encontró al microorganismo fueron la secreción de herida operatoria y el aspirado endotraqueal. Este hallazgo permite deducir 
que la bacteria puede desplazarse a diversos órganos y tejidos y no solo a las vías urinarias cuando las condiciones le favorecen ${ }^{(12)}$.

El $57 \%$ de nuestros pacientes recibieron tratamiento antibiótico anterior en el establecimiento de procedencia (HNGAI), y los 9 del Hospital Dos de Mayo ${ }^{(8)}$ recibieron también antibióticos previamente. Esta situación constituye un factor de riesgo importante para la aparición de infecciones multirresistentes, como se reportó en el Hospital Universitario de Bogotá el $2011^{(13)}$.

La mortalidad en el nuestro estudio fue de $28,6 \%$ ( 2 de 7 pacientes), que no es muy alta si se considera que en otros reportes varía del $55 \%$ al $68 \%{ }^{\left({ }^{6}\right)}$ y en el Instituto Nacional de Salud es del 47 al $68 \%$ (14). Esta mortalidad estuvo asociada a la coexistencia de hepatopatía crónica en nuestros dos fallecidos que no tuvieron mejoría pese al tratamiento con tres antibióticos: en el primer caso se empleó vancomicina, meropenem y colistiva, y en el segundo caso utilizaron imipenem, ciprofloxacina, y ceftriaxona. Cabe mencionar que no se existía una hepatopatía en los pacientes que sobrevivieron.

La terapia antibiótica recibida en los casos no mortales fue variada, y solo en uno se prescribió amicacina (según sensibilidad del antibiograma), lo que nos indica que no existe un protocolo consensuado ni programa de optimización sobre el manejo antibiótico en nuestro hospital, lo que constituye una tarea pendiente que debe laborarse a la brevedad, en nuestro caso, por la instancia competente que es Instituto de Evaluación de Tecnologías en Salud e Investigación (IETSI) EsSalud. Al respecto, Salgado ${ }^{(15)}$ se refiere a los carbapenémicos como "los elegidos" para una base de terapia combinada con las polimixinas, fosfomicina o la gentamicina/ aminoglucósido, con resultados relativamente buenos tanto in vitro como in vivo.

En todos los pacientes, los mecanismos de resistencia bacteriana encontrados fueron la producción de carbapenemasas tipo MBL y la de betalactamasas tipo BLEE. Esto tuvo relevancia por las características clínicas halladas y la mortalidad encontrada, ya que este tipo de resistencias nos deja con escasas alternativas terapéuticas viables $^{(16)}$.

El patrón de resistencia a los carbapenémicos de la Klebsiella pneumoniae hallado se caracterizó por resistencia al meropenem en todos los casos y sensibilidades reducidas al imipenem y al ertapenem. Ante esta multiresistencia progresiva y en aumento, algunos autores recomiendan la terapia antibiótica combinada, en la que se agrega colistina o fosmicina a altas dosis, que se puede modificar según la CIM del antibiograma ${ }^{(17)}$.

La susceptibilidad de las cepas encontradas a los antibióticos no carbapenémicos (22 elegidos en total) se caracterizó por una sensibilidad total a la amicacina y a colistina, y en segundo lugar a tigeciclina, seguido de levofloxacina y nitrofurantoína. Esta sensibilidad total a la amicacina fue reportada en el Hospital Dos de Mayo de Lima, pero no sería un patrón fenotípico usual ${ }^{(8)}$. En contraste, en el Reino Unido se observó una resistencia emergente (asociada al gen RmtF) a los aminoglucósidos en $14 \%$ de los casos estudiados ${ }^{(18)}$. Otro hallazgo importante en este trabajo es haber encontrado $100 \%$ de resistencia de cepas de Klebsiella pneumoniae a la ampicilina, aztreonam, cefepima, cefoxitina, cefuroxima, gentamicina, piperazilina/tazobactam, tobramicina, y sulfametoxazol/ trimetoprima. Por esta razón, la tendencia actual es usar combinaciones relativamente nuevas de antibióticos, como se hizo en un hospital colombiano, que asoció imipenem con ciprofloxacina en un brote de Klebsiella pneumoniae NDM-1 en una $\mathrm{UCl}$ neonatal ${ }^{(6)}$. Asimismo, una asociación antibiótica actual es la de una cefalosporina (cefotaxima o ceftriaxona) y un carbapenémico ${ }^{(19)}$, y otras asociaciones clásicas como un carbapenémico con colistina o tigeciclinaque muestra una reducción de la mortalidad por Klebsiella pneumoniae ${ }^{(20)}$.

El proceso de detección del mecanismo de resistencia involucrado se prolongó, usualmente, entre 5 y 7 días en cada muestra, a las cuales, además, se les tuvo que realizar pruebas complementarias, algunas veces fuera del hospital como el caso de la PCR. Para ello requerimos de coordinaciones transversales, el uso de protocolos consensuados y actualizados entre las redes de atención; asimismo, contar con personal de laboratorio entrenado y comprometido.

Será conveniente realizar otros trabajos de investigación que podrían abarcar el componente prospectivo para conocer la evolución de la enfermedad, la existencia de portadores sanos de Klebsiella pneumoniae y proseguir con las medidas y estrategias generales de control y prevención de las infecciones asociadas a la atención de salud. Además, continuar con la realización de vigilancia y control epidemiológico de la IAAS y seguir con la identificación de cepas productoras de carbapenemasas, ya que se diseminan fuera de instituciones de salud y llegan a la comunidad.

Asimismo, se recomienda la elaboración de los protocolos validados de manejo de las infecciones severas por enterobacterias y otros bacilos gramnegativos, y alinearse a las recomendaciones de la OMS relativas a usar la herramienta informática WHONET- SatScan en la detección de brotes epidémicos en tiempo real para lo que se debe asignar a personal competente en esta labor.

Contribuciones de autoría: El Dr. Víctor Buendía Sotelo ha participado en la concepción del artículo, recolección de datos, análisis e interpretación, redacción y aprobación de 
la versión final. Además, la Dra. Shirley Morales Gutiérrez, el TM Jaime Coca Núñez y el TM Lenin Santos Varas contribuyeron en la redacción del protocolo, y análisis de datos. El estadístico José A. Soriano Toyama contribuyó en la confección de tablas y el análisis estadístico. La Dra. Shirley Morales Gutiérrez, el TM Jaime Coca Núñez y el TM Lenin Santos Varas intervinieron el análisis e interpretación de datos, redacción final de estudio. El Dr. Juan Guanira nos asesoró en la versión final y revisión crítica del artículo.

Fuente de financiamiento: Este artículo ha sido financiado por los autores.

Conflictos de interés: Los autores declaran no tener ningún conflicto de interés.

\section{REFERENCIAS BIBLIOGRÁFICAS}

1. Cardo D, Dennehy PH, Halverson P, Fishman N, Kohn M, Murphy $\mathrm{CL}$, et al. Moving toward elimination of healthcare-associated infections: a call to action. Infect Control Hosp Epidemiol. 2010; 31(11): 1101-5.

2. Pujol M, Limón E. Epidemiología general de las infecciones nosocomiales. Sistemas y programas de vigilancia. Enferm Infecc Microbiol Clin. 2013; 31(2): 108-13.

3. Unahualekhaka A. Epidemiología de las infecciones asociadas a la atención de salud. 2018. Disponible en: https://www.theific.org/ wp-content/uploads/2014/08/Spanish_ch3_PRESS.pdf

4. Yong D, Toleman MA, Giske GC, Cho HS, Sundman K, Lee K, et al. Characterization of a new metallo-beta-lactamasa gene, bla (NDM1), and a novel erythromicin esterase gene carried on a unique genetic structure in Klebsiella pneumoniae sequence type 14 from India. Antimicrob Agents Chemoter. 2009; 53(12): 5046-54.

5. Pasterán F, Albornoz E, Faccone D, Gómez S, Valenzuela C, Morales $M$, et al. Emergence of NDM-1-producing Klebsiella pneumoniae in Guatemala. J Antimicrob Chemoter. 2012; 67(7): 1795-7.

6. Escobar Pérez JA, Olarte Escobar NM, Castro-Cardozo B, Valderrama Márquez IA, Garzón Aguilar MI, Martínez de la Barrera $\mathrm{L}$, et al. Outbreak of NDM-1 producing Klebsiella pneumoniae in a Neonatal Unit in Colombia. Antimicrob Agents Chemoter. 2013; 57(4): 1957-60.

7. Espinoza JC. Presencia de casos de Klebsiella pneumoniae y/o Staphylococus aureus meticilino resistentes en pacientes del servicio de medicina del Hospital Antonio Lorena-Cusco. Alerta Epidemiológica $\mathrm{N}^{\circ}$ 001-2016. Disponible en: http:// www.diresacusco.gob.pe/inteligencia/epidemiologia/alertas/ alertas2016/ALERTA\%20\%20EPIDEMIOLOGICA\%20001-2016.pdf

8. Resurrección-Delgado C, Montenegro-Idrogo JJ, Chiappe-Gonzalez A, Vargas-Gonzales R, Cucho-Espinoza C, Mamani-Condori DH, et al. Klebsiella pneumoniae Nueva Delhi metalo-betalactamasa en el Hospital Nacional Dos de Mayo, Lima. Rev Perú Med Exp Salud Pública. 2017; 34(2): 261-7.

9. Hospital Clínica San Isidro Labrador. Reporte de referencias recibidas en el Hospital II San Isidro Labrador de febrero a mayo 2018. EsSalud; 2018.

10. Hospital Clínica San Isidro Labrador. Reporte anual mensualizado de incidencia de microrganismos productores de BLEE y carbapenemasas. Servicio de Patología Clínica-EsSalud; 2017.

11. Carrasquer Pirla T, Solano Luque M, Martínez Martínez J, Oliver Caldes A, Clemos Matamoros S. Infección urinaria por Klebsiella pneumoniae productora de carbapenemasas OXA-48: experiencia de 4 casos. Rev Clin Esp. 2015; 215(Espec Congr): 1088.

12. Bush LM. Infecciones por Klebsiella, Enterobacter y Serratia. Manual MSD. Disponible en: https://www.msdmanuals.com/espe/hogar/infecciones/infecciones-bacterianas/infecciones-porklebsiella-,-enterobacter-y-serratia

13. Alvarez C, Linares C, Gomez CH, Al L, Gil F, Puerta J, et al. Factores de riesgo para infección o colonización por Klebsiella pneumoniae resistente a carbapenémicos en un hospital Universitario de tercer nivel, Bogotá, 2009-2010. VIII Encuentro Nacional de Investigación en Enfermedades Infecciosas. Armenia; 2012.

14. Instituto Nacional de Salud. Protocolo para detección de KPC en enterobacterias. Lima: Centro Nacional de Salud Pública, Instituto Nacional de Salud. 2014.

15. Salgado P, Gilsanz F, Maseda E. Tratamiento de infecciones causadas por enterobacterias productoras de carbapenemasas. Rev Esp Quimioter. 2015; 28(Suppl. 1): 12-5.

16. Rodríguez-Noriega E, León-Garnica G, Petersen-Morfín S, PérezGómez HR, González Díaz-E, Morfín-Otero R. La evolución de la resistencia bacteriana en México, 1973-2013. Biomédica. 2014; 34(Suppl. 1): 181-90.

17. Martinez Sagasti F, González Gallego MA, Moneo González A. Monoterapia vs. terapia combinada en el tratamiento de infecciones por bacterias gramnegativas multiresistentes. Rev Esp Quimioter. 2016; 29(Suppl. 1): 43-6.

18. Hidalgo L, Hopkins KL, Gutierrez B, Ovejero CM, Shukla S, Douthwaite $\mathrm{S}$, et al. Association of the novel aminoglycoside resistance determinant RmtF with NDM carbapenemasa in India and UK. J Antimicrob Chemosther. 2013; 68(7): 1543-50.

19. Web MD. What is Klebsiella pneumoniae infection? [internet]. 2018. Disponible en: https://www.webmd.com/a-to-z-guides/ klebsiella-pneumoniae-infection\#1

20. Daikos GL, Tsaousi S, Tzouvelekis LS, Anyfantis I, Psichogiou M, Argyropoulou A, et al. Carbapenemase-producing Klebsiella pneumoniae bloodstream infections: lowering mortality by antibiotic combination schemes and the role of carbapenes. Antimicrob Agents Chemother. 2014; 58(4): 2322-8.

\section{Correspondencia:}

Victor Manuel Buendia Sotelo

Dirección: Av. Javier Prado 8466 Urb. Los portales, Ate. Lima - Perú.

Teléfono: 993476851

Correo Electrónico: victor_buendia1949@yahoo.com

Recibido: 08 de abril de 2019

Evaluado: 01 de julio de 2019

Aprobado: 24 de julio de 2019

๑ La revista. Publicado por Universidad de San Martín de Porres, Perú.

(cc) Br Licencia de Creative Commons Artículo en acceso abierto bajo términos de Licencia Creative Commons Atribución 4.0 Internacional. (http://creativecommons.org/licenses/by/4.0/)

\section{ORCID iDs}

Víctor Buendía Sotelo

Shirley Morales Gutiérrez

Jaime Coca Núñez

Lenin Santos Varas
(ㄱ) https://orcid.org/0000-0001-8405-6105 (1) https://orcid.org/0000-0002-9702-9904 (1) https://orcid.org/0000-0002-4878-8234

근 https://orcid.org/0000-0001-5633-9618 